\title{
Determinantes de utilização do cuidado pré-natal entre famílias de baixa renda no Estado da Paraíba, Brasil
}

\author{
Determinants of prenatal care utilization among low income familes in Paraíba \\ State, Brazil
}

José Rodrigues Filho*, Walfredo da Costa**, Glaucia Maria de Luna leno**

\begin{abstract}
RODRIGUES FILHO, J. et al. Determinantes de utilização do cuidado pré-natal entre famílias de baixa renda no Estado da Paraíba, Brasil. Rev. Saúde Pública, 28: 284-9, 1994. Analisaram-se os determinantes de utilização da assistência pré-natal, entre famílias de baixa renda. Dados foram coletados de todas as mães com residência permanente no Município de Caaporã, no Estado da Paraíba, Brasil, com filhos até cinco anos de idade na data da entrevista. Através da estatística descritiva e multivariada, analisaram-se os diferenciais de utilização dos serviços e os efeitos de algumas variáveis sociodemográficas sobre o uso do cuidado pré-natal.
\end{abstract}

Descritores: Cuidado pré-natal, utilização. Fatores socioeconômicos.

\section{Introdução}

Apesar das evidências de que o cuidado prénatal contribui para a melhoria dos resultados da gravidez, muitas mulheres nos países subdesenvolvidos e em desenvolvimento são carentes de assistência pré-natal ${ }^{4,5,7,13}$. Já foi demonstrado que as complicações na gravidez e no parto são as principais causas de morte entre as mulheres em período de reprodução (15-44 anos), o que vem fortalecer a relação entre a mortalidade/morbidade materna e a ausência total ou inadequada do cuidado pré-natal ${ }^{9}$. Embora um dos principais objetivos dos programas de assistência primária de saúde nesses países tenha sido o de aumentar o número de consultas para mulheres gestantes, a assistência inadequada entre mulheres de baixa renda ainda é registrada $9,14,15$.

Além disto, informações sobre o padrão de assistência pré-natal nos países em desenvivolvimento são quase inexistentes, apesar da crescente utilização dos serviços de saúde pelas populações de baixa renda, nos últimos anos.

No Brasil, por exemplo, estratégias de assistência primária de saúde destinadas a melhorar o cuidado pré-natal, especialmente nas áreas mais po-

\footnotetext{
* Departamento de Administração da Universidade Federal da Paraf́a - João Pessoa, PB - Brasil

** Departamento de Promoção da Saúde da Universidade Federal da Paraíba - João Pessoa, PB - Brasil
}

Separatas/Reprints: J.Rodrigues Filho - Av. Silvino Lopes, 425/304 - 58039-190 - João Pessoa, PB Brasil bres, são ineficientes. Alguns estudos têm demonstrado que, nas áreas mais desenvolvidas do país, tem havido melhorias na assistência pré-natal. Em São Paulo, por exemplo, $30 \%$ das mulheres de baixa renda não receberam assistência pré-natal em 1981, porém informações mais recentes indicam que, em algumas áreas, apenas $15 \%$ não estão tendo esta assistência ${ }^{1}$.

Contudo, informações sobre o cuidado prénatal na região Nordeste são pouco conhecidas. Em 1986, a Sociedade Civil Bem-Estar Familiar no Brasil (BENFAM) ${ }^{2}$ constatou que "somente 55 $\%$ dos nascimentos ocorridos nos últimos cinco anos tiveram controle pré-natal". Além disto, há informações de que a adequada assistência prénatal pode reduzir as complicações da gravidez e o número de crianças de baixo peso ao nascer. Pesquisa realizada no interior do Ceará, por exemplo, mostrou que a assistência pré-natal reduziu as chances de mães perderem seus filhos no período neo-natal ${ }^{10}$. A pesquisa da BENFAM ${ }^{2}$ demonstrou ainda que "no Brasil, em geral, o parto domiciliar está associado a uma baixa frequêência de atendimento no pré-natal e a uma assistência inadequada no momento do nascimento, aumentando os riscos da mortalidade perinatal".

Tem sido mostrado que tanto no Brasil como em outros países em desenvolvimento variáveis demográficas e socioeconômicas estão associadas com a utilização dos serviços relacionados à assistência pré-natal ${ }^{2,8}$. Portanto, o propósito do presente trabalho é o de analisar os determinantes de utilização da assistência pré-natal entre famílias de baixa renda e as características das mulheres resi- 
dentes no Município de Caaporã, Estado da Paraíba.

\section{Metodologia}

O presente trabalho foi realizado com dados provenientes do Município de Caaporã, situado na microregião do Litoral Paraibano e ocupando uma área de $100 \mathrm{~km}^{2}$. Caaporã em língua indígena significa "Boca da Mata" (" Caa " = Boca e "Porã " = Mata). A população residente, com base no censo de 1990 , é de 15.000 habitantes, sendo a composição etária da população predominantemente jovem, com $45 \%$ residindo na zona rural.

A cana-de-açúcar ocupa a maior parte do solo do município, embora mereça destaque a plantação de coco, lavoura de subsistência e inhame. A renda familiar é relativamente baixa, já que a maior parte da população recebe o salário mínimo. Os desempregados, em número significativo, têm renda incerta e, muitas vezes, inferior ao salário mínimo, já que se empregam na cultura cíclica (cana de açúcar) e sobrevivem da atividade pesqueira. A maioria da população reside em casas de um ou dois quartos, construídas de barro, incluindo o piso. A ocupação média de cada residência é de quatro pessoas. A população recebe assistência médica de um posto de saúde do município e da maternidade local, porém muitas pessoas se dirigem a João Pessoa a cerca de $70 \mathrm{~km}$ de Caaporã.

Foram coletados dados de todas as mães com residência permanente no município e com filhos até 5 anos de idade na data da entrevista. Durante um período de 6 meses (novembro 1992 a abril 1993), 793 mães foram entrevistadas, em suas residências, por três estudantes do sexo feminino, cujo trabalho era semanalmente supervisionado pelos autores da presente pesquisa.

O instrumento de coleta de dados foi um questionário de 72 perguntas, testado pelos autores durante as duas primeiras semanas do inquérito. $\mathrm{O}$ questionário foi desenhado com o propósito de obter informações sobre o cuidado pré-natal, número de filhos, educação, idade, tipo de moradia, mortalidade infantil, além de outros dados socioeconômicos e as práticas de amamentação e de contracepção. Para o propósito do presente trabalho, 24 mulheres foram excluídas por conta do preenchimento incompleto de questionários. Assim sendo, foram consideradas apenas informações inerentes a 769 mães.

Sendo o objetivo da presente análise examinar os efeitos de características demográficas e socioeconômicas sobre a utilização da assistência prénatal (consultas médicas), foram consideradas as variáveis residência, número de pessoas na família, nível educacional, idade, e tipo de moradia. Para a análise dos dados recorreu-se à estatística descritiva e a tabulações cruzadas, com o propósito de enfatizar as variáveis associadas com a utilização da assistência pré-natal. Além disto, a análise multivariada foi utilizada para aprofundar o estudo da associação existente entre essas variáveis e a utilização da assistência pré-natal.

Uma vez que a variável dependente (assistência pré-natal) é binária, codificada zero (nenhuma consulta) ou um (uma ou mais consultas), decidiuse não utilizar a regressão linear. Consequientemente, a regressão logística foi usada para estimar os coeficientes de todas as variáveis independentes (incluindo-se medidas dicotômicas).

A análise com logit ${ }^{3,6,11}$ pressupõe que existe uma tendência de se visitar o médico, que é uma função linear das variáveis exploratórias. Pressupondo-se a função densidade, então a probabilidade de ocorrência de um evento, ou seja, de se usar o cuidado pré-natal $(\mathrm{P})$ é

$$
P=\frac{1}{1+e^{-Y}}
$$

Esta função tem as propriedades desejadas de serem limitadas pelos valores de zero (nenhuma consulta) ou um (uma ou mais consultas).

$$
Y=\ell n \frac{P}{1-P}=B_{0}+B_{1} X_{1}+B_{2} X_{2}+\ldots+B k X_{k}+\Sigma
$$

Onde:

$\ell \mathrm{n}=$ logaritmo natural

$\mathrm{X}_{\mathrm{i}}$ expressa uma variável independente

$B_{\mathrm{i}}$ expressa um coeficiente de regressão

$\sum$ é o termo dos erros

Neste caso, a equação do modelo formulado é estimada com a complexa técnica do modelo logit, denominada de máxima verossimilhança, o qual foi utilizado para estimar a assistência prénatal prestada a todas as mães residentes no município, bem como as residentes apenas na zona urbana.

\section{Resultados}

A Tabela 1 apresenta a distribuição de variáveis inerentes às mães, residência e condições socioeconômicas da população estudada. 
Tabela 1. Características individual e familiar de 769 mulheres que tiveram filhos nos últimos cinco anos anteriores ao inquérito, Município de Caaporă, Paraíba, 1993.

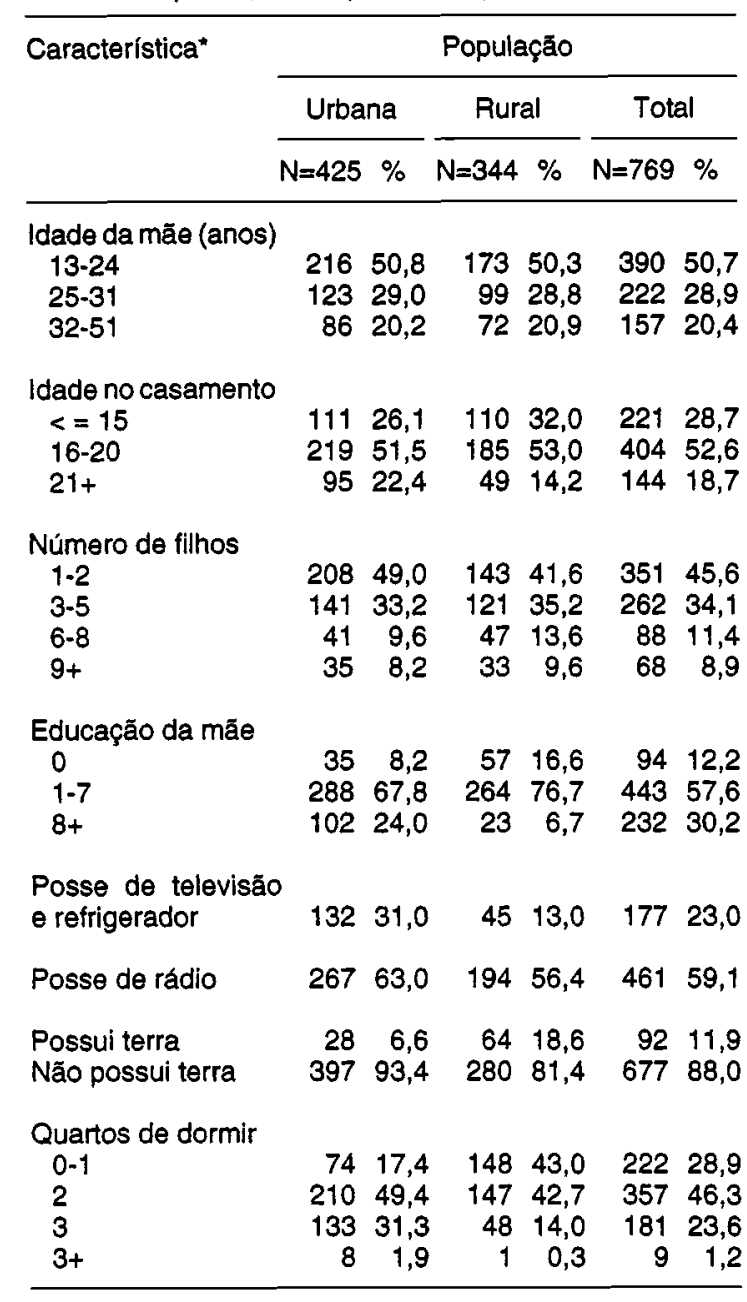

* Características na data do inquérito.

Observa-se que $55 \%$ das mães vivem na zona urbana, em condições precárias. Apenas $23 \%$ das residências do município possuem geladeira e televisão, sendo que o percentual da zona urbana é mais de duas vezes o da zona rural $(31 \%$ e $13 \%$, respectivamente). Muitas das casas são construídas de barro, com paredes sem revestimento e sem privadas apropriadas.

A Tabela 1 mostra a idade no casamento das mulheres em Caaporã: mais de $80 \%$ se casaram em idade de, no máximo, 20 anos, sendo que $46 \%$ tiveram de um a dois filhos. O percentual de mulheres com maior número de filhos é mais elevado na zona rural. Enquanto apenas $8,2 \%$ tiveram 6 a 8 filhos na zona urbana, na zona rural o percentual se eleva para 13,6\%. O nível educacional é baixo, já que $12 \%$ delas nunca freqüentaram escola.

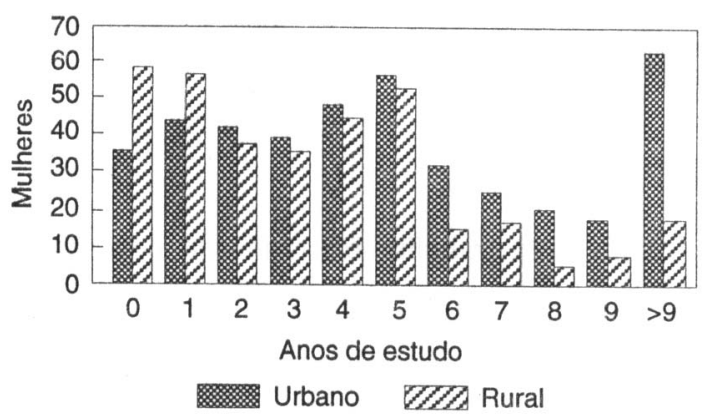

Figura - Escolaridade de mulheres com filhos de 0.5 anos de idade (urbana/rural) - Município de Caaporă, Paraiba, 1993.

Além disto, $58 \%$ de todas as mulheres estudadas atingiram apenas 7 anos de escolaridade. Como mostra a Figura, o nível educacional das mulheres residentes na zona rural é bastante inferior ao das residentes na zona urbana. Vê-se que tanto o número como o percentual de mulheres residentes na zona rural, sem nenhuma instrução, é o dobro do registrado na zona urbana $(16,6 \%$ e $8,2 \%$, respectivamente). Portanto, além de ser maior o número de mulheres sem nenhuma instrução na zona rural, este número decresce em relação às mulheres da zona urbana, na medida em que aumenta o nível de escolaridade (anos de estudo). Apesar de residirem numa das áreas de terras mais férteis da Paraíba, $81 \%$ das famílias da zona rural não possuem terra.

A Tabela 2 apresenta informaçōes sobre o uso de assistência pré-natal. Observa-se que $30 \%$ das mães não receberam nenhuma assistência pré-

Tabela 2 - Mulheres que tiveram filhos nos últimos cinco anos anteriores ao inquérito, segundo o tipo de assistência pré-natal, Município de Caaporã, Paraíba, 1993.

\begin{tabular}{|c|c|c|c|c|c|}
\hline \multirow{3}{*}{$\begin{array}{l}\text { Tipo de assistência } \\
\text { pré-natal }\end{array}$} & \multicolumn{5}{|c|}{ População } \\
\hline & \multicolumn{2}{|c|}{ Urbana } & Rural & \multicolumn{2}{|c|}{ Total } \\
\hline & $N=425$ & $\%$ & $N=344 \%$ & $N=769$ & $\%$ \\
\hline \multicolumn{6}{|l|}{ Número de visitas } \\
\hline $\begin{array}{l}\text { Nenhuma } \\
<=4 \\
>=5\end{array}$ & $\begin{array}{r}83 \\
96 \\
246\end{array}$ & $\begin{array}{l}19,5 \\
22,6 \\
57,9\end{array}$ & $\begin{array}{rr}143 & 41,6 \\
83 & 24,1 \\
118 & 34,3\end{array}$ & $\begin{array}{l}226 \\
179 \\
364\end{array}$ & $\begin{array}{l}29,4 \\
23,3 \\
47,3\end{array}$ \\
\hline \multicolumn{6}{|l|}{ Início da assistência } \\
\hline $\begin{array}{l}\text { Nenhuma } \\
1^{8} \text { trimestre } \\
\text { Depois }\end{array}$ & $\begin{array}{r}83 \\
228 \\
114\end{array}$ & $\begin{array}{l}19,5 \\
53,7 \\
26,8\end{array}$ & $\begin{array}{r}14341,6 \\
11433,1 \\
8725,3\end{array}$ & $\begin{array}{l}226 \\
342 \\
201\end{array}$ & $\begin{array}{l}29,4 \\
44,5 \\
26,1\end{array}$ \\
\hline \multicolumn{6}{|l|}{ Escala de assistência } \\
\hline $\begin{array}{l}\text { Nenhuma } \\
\text { Alguma }\end{array}$ & $\begin{array}{r}83 \\
342\end{array}$ & $\begin{array}{l}19,5 \\
80,5\end{array}$ & $\begin{array}{l}14341,6 \\
20158,4\end{array}$ & $\begin{array}{l}226 \\
543\end{array}$ & $\begin{array}{l}29,4 \\
70,6\end{array}$ \\
\hline
\end{tabular}


Tabela 3 - Diferencial de utilização do cuidado pré-natal entre mulheres que tiveram filhos nos últimos cinco anos anteriores ao inquérito, segundo variáveis de residência e educação da mãe, Municipio de Caaporã, Paraíba, 1993.

\begin{tabular}{|c|c|c|c|c|c|c|c|c|}
\hline \multirow[b]{2}{*}{ Variável } & \multicolumn{3}{|c|}{ Cuidado Pré-natal } & \multicolumn{5}{|c|}{ Atendimento ao parto } \\
\hline & Nenhuma & $\%$ & Alguma & $\%$ & Médico & $\%$ & Parteira & $\%$ \\
\hline \multicolumn{9}{|l|}{ Residência } \\
\hline $\begin{array}{l}\text { Urbana } \\
\text { Rural }\end{array}$ & $\begin{array}{c}83 \\
143\end{array}$ & $\begin{array}{l}(19,5) \\
(41,6)\end{array}$ & $\begin{array}{l}342 \\
201\end{array}$ & $\begin{array}{l}(80,5) \\
(58,4)\end{array}$ & $\begin{array}{l}218 \\
122\end{array}$ & $\begin{array}{l}(51,3) \\
(35,5)\end{array}$ & $\begin{array}{l}207 \\
222\end{array}$ & $\begin{array}{l}(48,7) \\
(64,5)\end{array}$ \\
\hline \multicolumn{9}{|l|}{$\begin{array}{l}\text { Educação } \\
\text { da Mãe }\end{array}$} \\
\hline $\begin{array}{l}\text { Nenhuma } \\
1-7 \\
8+\text { anos }\end{array}$ & $\begin{array}{c}42 \\
176 \\
8\end{array}$ & $\begin{array}{c}(46,3) \\
(28,9) \\
(6,2)\end{array}$ & $\begin{array}{r}51 \\
370 \\
122\end{array}$ & $\begin{array}{l}(53,7) \\
(71,1) \\
(93,8)\end{array}$ & $\begin{array}{c}31 \\
235 \\
74\end{array}$ & $\begin{array}{c}(9,1) \\
(69,1) \\
(21,8)\end{array}$ & $\begin{array}{c}62 \\
311 \\
56\end{array}$ & $\begin{array}{l}(14,5) \\
(72,5) \\
(13,0)\end{array}$ \\
\hline
\end{tabular}

natal, enquanto que apenas $44 \%$ iniciaram as visitas do pré-natal nos primeiros meses da gravidez. Na zona rural, o percentual de mulheres sem assistência pré-natal é duas vezes maior do que na zona urbana $(41,6 \%$ e $19,5 \%$, respectivamente). Portanto, observa-se que é elevado o percentual de mães sem assistência pré-natal e, das que tiveram alguma assistência, apenas $34 \%$ das residentes na zona rural fizeram mais de 5 consultas durante a gravidez.

A Tabela 3 mostra a relação positiva entre 0 . nível educacional e a assistência pré-natal e a relação negativa entre o local de residência (zona rural) e a utilização de assistência pré-natal. Por outro lado, a Tabela 3 mostra que $51 \%$ das mães residentes na zona urbana tiveram partos assistidos pelo médico, enquanto que a maioria das mães residentes na zona rural tiveram partos assistidos por parteiras (65\%). A proporção de mães residentes na zona urbana, recebendo algum tipo de cuidado pré-natal, é de $81 \%$ contra $58 \%$ daquelas residentes na zona rural.

No presente modelo de utilização do cuidado pré-natal, através da regressão logística, incluímos as duas variáveis utilizadas na Tabela 3 para o cálculo do diferencial de utilização do cuidado pré-natal, bem como variáveis inerentes à idade da mãe no casamento, tamanho familiar e condições de habitação. No modelo apresentado na Tabela $4, X_{1}$ representa o número de anos de estudo de cada mãe; $\mathrm{X}_{2}$ é uma variável muda, que toma o valor de 1 (um) se existe a posse de rádio e 0 (zero) se não existe; $X_{3}$ representa o número de pessoas de cada família; $\mathrm{X}_{4}$ é o tipo de construção (tijolos, taipa, palha, e outros) tendo sido atribuído a esta variável valores de 1 a $6 ; \mathrm{X}_{5}$ representa o tipo de privada (com descarga, sem descarga, casinha e outros) tendo sido atribuído valores de 1 a 4 a esta variável; $\mathrm{X}_{6}$ representa a idade da mãe no casa- mento; $\mathrm{X}_{7}$ e $\mathrm{X}_{8}$ são variáveis mudas, as quais foram atribuídas valores zero e um.

Assim, a Tabela 4 apresenta as estimativas do modelo logit e os efeitos das variáveis sobre a utilização da assistência pré-natal. Com exceção da variável referente à idade da mãe no casamento, as demais tiveram efeito significativo sobre o cuidado pré-natal. Portanto, quanto mais elevado for o nível educacional da mãe, maior é a utilização de serviços de pré-natal. Similarmente, há uma associação positiva entre residência (zona urbana) e assistência pré-natal. A existência de privadas mais adequadas e de residências construídas de tijolos têm um efeito positivo sobre a utilização do cuidado pré-natal. Em resumo, melhores condições de habitação são positivamente associadas com a assistência pré-natal. Por outro lado, o tamanho da família é negativamente associado com a assistência pré-natal, ou seja, mães com menos filhos tendem a demandar mais assistência.

\section{Discussão}

Os resultados apresentados mostram que há insuficiência de utilização dos serviços de assistência pré-natal, tanto de mães residentes na zona urbana quanto rural. Observou-se que $41,6 \%$ das mães residentes na zona rural não receberam nenhuma assistência, ao passo que apenas $34 \%$ fizeram mais de quatro visitas durante a gravidez. $\mathrm{Na}$ zona urbana $80 \%$ das mães receberam algum atendimento de pré-natal e $58 \%$ fizeram mais de quatro visitas.

As variáveis de características socioeconômicas examinadas mostraram estar associadas com a utilização de assistência pré-natal. A análise demonstrou que o nível educacional e $o$ local de residência (zona urbana) contribuíram para aumentar o número de visitas realizadas. 
Tabela 4 - Estimativa dos parâmetros e erro padrão do modelo Logit

\begin{tabular}{|c|c|c|c|c|}
\hline \multirow[b]{2}{*}{ Variável } & \multicolumn{2}{|c|}{ População Total } & \multicolumn{2}{|c|}{ População Urbana } \\
\hline & Coef. & SE & Coef. & SE \\
\hline $\begin{array}{l}\text { Constante } \\
X_{1} \text { Educação } \\
X_{2} \text { Posse de rádio } \\
X_{3} \text { Tamanho da familia } \\
X_{4} \text { Tipo de construção } \\
X_{5} \text { Privada } \\
X_{6} \text { Idade no casamento } \\
X_{7} \text { Residência } \\
X_{8} \text { Água encanada } \\
n\end{array}$ & $\begin{array}{c}0,3140 \\
0,1518^{\star} \\
-0,5748^{\star} \\
-0,0703^{\star *} \\
-0,3216^{\star * \star} \\
-0,2384^{\star \star} \\
0,0495 \\
0,8114 \\
- \\
769\end{array}$ & $\begin{array}{c}0,6433 \\
0,0345 \\
0,1795 \\
0,0291 \\
0,1518 \\
0,0892 \\
0,0275 \\
0,1804 \\
\cdot\end{array}$ & $\begin{array}{c}1,7187^{\star * *} \\
0,0881^{* * *} \\
0,5692^{\star *} \\
-0,1441^{*} \\
- \\
-0,4601^{*} \\
0,0827 \\
- \\
0,6464^{* *} \\
425\end{array}$ & $\begin{array}{c}0,9555 \\
0,0496 \\
0,2868 \\
0,0418 \\
- \\
0,1470 \\
0,0460 \\
- \\
0,3125\end{array}$ \\
\hline
\end{tabular}

* Significante para $p<0,001 ;{ }^{* \star} p<0,01 ;{ }^{* \star *} p<0,05$

Esses resultados são similares aos de estudos semelhantes realizados em outros países ${ }^{8}$. Observou-se ainda que mães com maior número de filhos, residindo em condições mais precárias, tendem a diminuir a utilização de assistência prénatal, enquanto que a idade da mãe no casamento demonstrou não ser um estimador significante de utilização dos serviços de assistência ao pré-natal. Conclui-se que quanto mais elevado forem as condições socioeconômicas das mães mais intenso será o uso dos serviços relacionados ao cuidado pré-natal.

Para reduzir os diferenciais de utilização do cuidado pré-natal, barreiras econômicas e não econômicas devem ser consideradas. Tem sido enfatizado que as características do sistema de saúde, por exemplo, desempenham papel de grande importância para entravar o acesso de grupos específicos aos cuidados do pré-natal.

Assim sendo, a inexistência de um sistema de saúde compreensivo no Brasil em geral e na Paraíba, em particular, tem restringido a provisão de serviços de forma acessível e gratuito a uma grande parte da população, sobretudo aqueles que vivem abaixo da linha de pobreza. Como se sabe, o sistema de saúde do país é ineficiente, de elevado custo e orientado para concentrar recursos no subsistema curativo e nas áreas economicamente privilegiadas. Como já foi dito, a metodologia de alocação de recursos de saúde no Brasil é obsoleta e orientada para aumentar as disparidades entre regiões urbanas e rurais ${ }^{12}$.

As melhorias de acesso aos serviços de saúde vão depender de um profundo redirecionamento das políticas e programas de saúde, capazes de incorporar uma assistência primária de saúde, de modo que a população receba, a partir dela, assistência integral e básica de saúde. A assistência primária de saúde é de grande im- portância não só nos países desenvolvidos como nos países pobres.

Além disto, uma melhoria educacional da população e uma orientação das mães, através de programas de planejamento familiar, entre outras medidas setoriais e estruturais, podem contribuir para o desenvolvimento econômico, redução e alívio da pobreza.

Seria importante que os resultados do presente estudo fossem considerados no planejamento dos serviços de saúde do Município de Caaporã, no sentido de que as mulheres daquela região recebam assistência mais adequada, a partir da melhoria dos serviços existentes e da implementação dos serviços de saúde na zona rural para lhes garantir acesso aos programas de assistência pré-natal.

Muitas questões sobre o sistema brasileiro de saúde não foram ainda respondidas. Apesar das limitações do presente estudo, observou-se que os problemas de acesso e as deficiências do atendimento pré-natal são aparentes. Neste sentido, é que se recomenda a realização de outras pesquisas para explicitar outros fatores não considerados no presente trabalho e que possam ter influência na utilização da assistência materno-infantil no $\mathrm{Mu}$ nicípio de Caaporã.

Para alcançar uma provisão de assistência de saúde para a maioria da população brasileira, decisões políticas devem ser direcionadas para uma assistência integral, reforçando o nível primário de saúde, de modo que o processo de alocação de recursos de saúde seja baseado nas necessidades da população e não nos interesses privados ancorados no setor saúde. Com a reforma sanitária em andamento, possibilitando o controle social, espera-se que mais cedo ou mais tarde as milhares de mulheres dessasistidas do país possam receber o mínimo de cuidado necessário para evitar riscos e complicações da gravidez. 


\section{Agradecimentos}

À Universidade Federal da Paraíba pelas facilidades oferecidas; à Universidade de Nagasaki (Japão) pelo apoio financeiro e ao Sindicato de Trabalhadores Rurais de Caaporã pelo apoio logístico.

RODRIGUES FILHO, J. et al. [Determinants of prenatal care utilization among low income families in Paraíba State, Brazil]. Rev. Saúde Pública, 28: 284-9, 1994. The determinants of prenatal care in the state of Paraíba, Brazil, are examined. A population of 769 rural and urban women resident in the municipality of Caaporã and who had had a child within the five years immediatly preceding the survey were interviewed. It was found that there are deficiencies in the provision of health services, especially in the rural area. While 80 percent of the $\mathrm{u}$ men resident in the urban area had some prenatal care, more than 40 percent of women in the rural area had received no prenatal care at all. Through cross tabulations and multivariate analysis, the study assesses the diferentials in the utilization or services and the net effect of sociodemographic variables on the use of prenatal care. It was found that there is a positive association between education and preganancy of use of prenatal care and a negative association between rural residence and the use of prenatal care.

Keywords: Prenatal care, utilization. Socioeconomic factors.

\section{Referências Bibliográficas}

1. BAILEY, P.; TSUI, A.O.; JANOWITZ, B.; DOMINIK, R.; ARAUUO, L. A study of infant mortality and causes of death in rural Nort-east Brazilian community. J. Biosoc. Sci., 22: 349-63, 1990.
2. BENFAM. Pesquisa Nacional sobre Saúde MaternoInfantil e Planejamento Familiar - Brasil,1986. Rio de Janeiro, 1987.

3. GLANTZ, S.A. \& SLINKER, B.L. Primer of applied regression and analysis of variance. New York, McGrawHill, 1990.

4. GORTMAKER, S.L. The effects of prenatal care upon the health of the newborn. Am. J. Public Health, 69: 653, 1979.

5. GREENBERT, R.S. The impact or prenatal care in different social groups. Am. J. Obstet. Gynecol., 145: 797, 1983.

6. GUJARATI, D.N. Basic econometrics. New York, McGrawHill, 1988.

7. HALL, M.H.; CHNG, P.K.; MACGILLIVRAY, I. Is routine antenatal care worthwhile? Lancet, 2: 78, 1980.

8. OBERMEYER, C.M. \& POTTER, J.E. Maternal health care utilization in Jordan: a study patterns and determinants. Stud. Fam. Plann., 22:177-87, 1991.

9. OKAFOR, C.G. Availability and use of services for maternal and child health care in rural Nigeria. Int. J. Gynecol. Obstet., 34: 331-46, 1980.

10. OSIS, J.J.D.; HARDY, E.; FAUNDES, A.; ALVES, G. Fatores associados à assistência pré-natal entre mulheres de baixa renda no Estado de São Paulo, Brasil. Rev. Saúde Pública, 27: 49-53, 1993.

11.PINDYCK, R.S. \& RUBINFELD, D.L. Econometrics models andeconomicforecasts. London, McGraw-Hill, 1981.

12. RODRIGUES FILHO, J. A distribuição dos recursos de saúde no Brasil: a administração da desigualdade. Rev. Adm. Empres., 27(3):52-7, 1987.

13. TOKAHATA, G.K.; DIGON, E.; MANN, L. Prenatal care and obstetric abonormalities. J. Chron. Dis., 25: 163 85,1973

14. WONG, E.L.; POPKIN, B.M.; GUILKEY, D.K.; AKING, J.S. Accessibility, quality of care and prenatal care use in the Philippines. Soc. Sci.Med. . 24: 927-44, 1987.

15. YANKAUER, A.; GROSS, K.G.; ROMEO SALVATORE, $M$. An evaluation of prenatal care and its relationship to social class and social disorganization. Am. J. Public Health, 43: 1001-10, 1953.

Recebido para publicação em 21.1.1994

Reapresentado em 26.4.1994

Aprovado para publicaçäo em 7.7.1994 